\title{
Kangaroo mother care: Mothers experiences post discharge from hospital
}

\author{
Opara PI*, Okorie EMC
}

Department of Paediatrics, University of Port Harcourt Teaching Hospital, Port Harcourt, Nigeria

\begin{abstract}
Background: Kangaroo Mother Care (KMC), a natural way of nursing newborns, where mothers use their own body temperature to keep their infants warm has proven to be as efficacious as incubator nursing for the care of low birth weight (LBW) infants and leads to their earlier discharge from hospital.

Objective: To explore mother's knowledge and experiences with KMC at home after discharge from hospital.

Method: Mothers who had practiced KMC and whose LBW infants had been discharged from the Special Care Baby Unit of a tertiary health institution in Southern Nigeria, were recruited into the study during follow up visits. Data collected using a simple structured questionnaire included, weight of babies on discharge, knowledge and practice of KMC at home, family support, benefits and problems encountered. Data were analyzed using SPSS 16.0.

Results: Forty-two mothers participated in the study. Mean weight of babies at discharge was $1.63 \pm$ $0.85 \mathrm{~kg}$. Mean duration of KMC/day at home was $3.25 \pm 2.85$ hours $(0.5-12$ hours). $16(38.1 \%)$ of babies were still receiving KMC. For those that had stopped, mean weight at termination was $2.45 \pm$ $0.95 \mathrm{~kg}$. The commonest reason was that baby became uncomfortable. $31(\mathbf{7 3 . 8 \%})$ of mothers didn't know that somebody else could assist with KMC. Commonest support received was help with domestic chores. No baby had problems with KMC at home. Over $95 \%$ of the mothers enjoyed KMC at home. Some concerns were inability to do domestic chores, sleep comfortably at night or give attention to other children while doing KMC.

Conclusion: Mothers are comfortable with KMC but need more information and support groups are advocated.
\end{abstract}

Keywords: Infants, Kangaroo mother care, Nursing, Neonatal.

\section{Introduction}

More than 20 million infants of low birth weight (LBW) are born worldwide annually with over $90 \%$ being born in developing countries [1]. This imposes a heavy burden on healthcare and social systems in developing countries as medical care of these infants is complex and requires costly infrastructure and highly skilled staff $[2,3]$. In order to combat some of these problems, as well as concerns of mother-infant separation, Kangaroo mother care (KMC), a simple method of care for LBW infants, was developed in 1978 by Edgar Rey, a Colombian Paediatrician [4].

In $\mathrm{KMC}$, newborns are attached to mothers' chests in skin to skin contact, wearing only a nappy and a hat in the upright position [4]. Mothers are thus, substitute incubators, providing warmth and nutrition for the low birth weight infant. KMC provides thermal care through continuous skin-to-skin contact, support for exclusive breastfeeding or other appropriate feeding, and allows for early recognition/response to illness [2]. $\mathrm{KMC}$ has been reported to facilitate early discharge from hospital and reduce neonatal morbidity and mortality [5-8]. Many studies report its benefits over incubator care and there is sufficient evidence to recommend its routine use in facilities for stable LBW infants [5,8,9]. KMC can be implemented in various facilities at different levels of care [10,11]. However, the potential effect of $\mathrm{KMC}$ is greatest in low-income countries, where facilities for care of LBW babies are limited with few, poorly equipped and understaffed neonatal care units [5]. In well-resourced neonatal care units, it still enhances mother- infant bonding and breast feeding and where neonatal care units are available but overwhelmed by demand as in our setting, KMC allows for rationalization of resources by freeing up incubators for sicker infants $[12,13]$

In places where the practice is established, KMC usually starts in hospital with an adaptation process. During adaptation and after discharge, mothers/caregivers attend a day clinic where they are trained, infants are monitored, and the mother/ caregiver becomes part of a support group or social peer network. Care is thereafter provided at home with follow up visits as needed [2].

Although KMC is not fully established in our centre in terms of support groups and peer networks, it has helped tremendously in the care of low birth weight infants. It is started in the hospital and continued at home with intermittent follow-up visits to monitor the baby's growth and development. The aim 
of this study was to explore experiences of mothers with KMC at home after discharge from hospital.

\section{Materials and Methods}

The study was carried out in the neonatal clinics of the University of Port Harcourt Teaching Hospital (UPTH). The hospital has a Special Care Baby Unit (SCBU) that caters for sick newborn infants born within and outside the hospital. It is the largest neonatal care unit in Port Harcourt metropolis and its environs and attracts referrals from in and around Rivers state where it is located. At the time of this report the unit had 10 functional incubators. The facility also has two breast feeding rooms with comfortable chairs for mothers and a mothers' room which is about a stone throw away where mothers stay during the period of their baby's admission in the unit. The demand for care of low birth weight infants is high and oftentimes the need for incubators overwhelms the available resources. The practice of $\mathrm{KMC}$ was introduced to the unit to help better utilization of resources.

Low birth weight infants who have been on incubator care are gradually weaned off to $\mathrm{KMC}$ as soon as they are haemodynamically stable. Mothers practice KMC intermittently while in hospital after they have had practice sessions with the nurses. This is done in the breastfeeding rooms where they sit upright with the baby strapped to the mother in the KMC position for one to three hours, once or twice every day until the infant is considered fit for discharge and the mother is also very comfortable with KMC. At discharge the mothers are counseled to continue KMC at home and are given appointments for follow up in the neonatal clinic weekly. This clinic is run by senior Pediatricians once every week and babies who had been nursed in the SCBU are followed up for various periods of time depending on the nature of morbidity and complications of the primary illness for which they were treated.

Mothers who had practiced KMC, while in hospital and whose LBW infants had been discharged from the SCBU at least four weeks prior to and within the duration of the study, were recruited into the study during follow up visits. Authors interviewed mothers who gave consent to be included in the study. Excluded were mothers who had multiple deliveries and who did not continue KMC after discharge. Data were collected using a simple structured interviewer administered questionnaire and included, weight of babies on discharge, knowledge and practice of KMC at home, family support, benefits and challenges encountered while doing $\mathrm{KMC}$ at home. Some data e.g., birth weight and weight on discharge were obtained from the babies' case notes. Data were analyzed using SPSS 16.0. Weights were measured at each visit and for those who had stopped $\mathrm{KMC}$, the weights taken in the clinic immediately following discontinuation of $\mathrm{KMC}$ were estimated to approximate weight at discontinuation of KMC.

\section{Results and Discussion}

Forty-two mothers participated in the study. The mean age of the mothers was $30.59 \pm 4.50$ years. All the infants were singleton. There were 23 males and 19 females giving a male to female ratio of 1.2:1. Mean gestational age at admission was $32.27 \pm 2.41$ weeks (29-39 weeks). The mean weight at commencement of $\mathrm{KMC}$ while on admission was $1.45 \pm 0.23$ $\mathrm{kg}$, while the mean weight at discharge was $1.60 \pm 0.18 \mathrm{~kg}$. Mean duration per day of KMC in hospital was $2.03 \pm 1.22$ hours. All the babies had received incubator care.

Mean duration of KMC per day at home was $3.25 \pm 2.85$ hours (0.5-12 hours). Type of support received by the mothers at home included help in performing household chores 24 (57.1\%), help with caring for other children 33 (78.2\%) and help with KMC 4 (9.5\%).

Some of the benefits the mothers derived from KMC included feeling more involved with baby's care 25 (59.5\%), and feeling closer to the baby $25(59.5 \%)$ in Table 1 .

Table 1. Benefits mothers derived from KMC.

\begin{tabular}{lll}
\hline Benefits & Number & Percent \\
\hline Felt closer to baby & 25 & 59.5 \\
\hline Felt more confident in caring for baby & 25 & 59.5 \\
\hline Baby seemed to grow faster & 15 & 35.7 \\
\hline Spent more time with baby & 15 & 35.7 \\
\hline Felt involved in baby's care & 13 & 31 \\
\hline Better lactation & 17 & 16.7 \\
\hline Reduced baby's contact with visitors & 24 & 60 \\
\hline Kept baby warm & 24 & 57.1 \\
\hline
\end{tabular}

All the babes were being exclusively breast fed at the time of this report. None of the mothers expressed any fears while doing $\mathrm{KMC}$ at home. Some of the problems highlighted with $\mathrm{KMC}$ at home included that it was time consuming 7 (16.7\%), and no time for domestic chores $5(11.9 \%)$ in Table 2 .

Table 2. Problems highlighted by mothers while doing KMC.

\begin{tabular}{lll}
\hline Problems & Number & Percent \\
\hline Time consuming & 7 & 16.7 \\
\hline No time for domestic chores & 5 & 11.9 \\
\hline Baby is hot and uncomfortable & 4 & 9.5 \\
\hline Less time for other children & 3 & 7.1 \\
\hline Too stressful & 3 & 7.1 \\
\hline None & 22 & 52.4 \\
\hline
\end{tabular}

Eleven (26.2\%) mothers knew that somebody else could assist with KMC. Of these, the grandmother $3(27 \%)$ and the father 1 $(9 \%)$ assisted while $7(64 \%)$ preferred to do it themselves.

Sixteen (38.1\%) babies were still on KMC while $26(61.9 \%)$ had stopped. For those who had stopped, mean weight at termination was $2.45 \pm 0.95 \mathrm{~kg}$. Reasons given included baby refusing KMC 21(80.8\%), mother felt it was okay $19(73.1 \%)$, 
and mother feeling uncomfortable with the position especially at night (Table 3 ).

Table 3. Reasons given by mothers for discontinuing KMC.

\begin{tabular}{lll}
\hline Reason & $\begin{array}{l}\text { Number } \\
(\mathbf{n = 2 6 )}\end{array}$ & Percent \\
\hline Baby refusing KMC position & 21 & 80.8 \\
\hline Mother felt it was Okay & 19 & 73.1 \\
\hline Baby had gained enough weight & 15 & 57.7 \\
\hline Mother felt uncomfortable sleeping at night & 3 & 11.5 \\
\hline $\begin{array}{l}\text { Mother did not have help with domestic } \\
\text { work }\end{array}$ & 2 & 7.7 \\
\hline
\end{tabular}

All the mothers would recommend $\mathrm{KMC}$ to other mothers with low birth weight babies and $31(73.8 \%)$ mothers agreed that if they had another low birth weight baby they would gladly do KMC again.

\section{Discussion}

All the mothers who participated in the study had commenced KMC in the hospital with the help of skilled health workers and infants had been discharged early with a mean discharge weight less than $1800 \mathrm{~g}$ which was the routine discharge weight of stable low birth weight infants in our centre prior to the introduction of KMC. One of the components of KMC is early discharge. Shorter length of hospital stay in KMC infants has been reported from both low and high-income countries $[3,13,14]$. This is particularly important in low income countries as it leads to reduction in cost of care both for the health facility and the care givers who in some cases like in our center pay out of pocket for these services.

Most mothers were comfortable with KMC at home although there were variations in the duration per day. This is probably attributable to other activities mothers have to engage in during the day. However, KMC has been shown to have positive physiological, psychosocial, and developmental effects whether provided for shorter or longer periods [15].

Most of the benefits of KMC reported in this study, have been reported by other authors [10,16,17]. The World Health Organization (WHO) describes KMC as "an effective way to meet baby's needs for warmth, breastfeeding, protection from infection, stimulation, safety and love [18] Reduced contact with visitors was of particular importance to our mothers as they felt they were protecting their delicate infants from the outside world. Reports have shown that KMC substantially reduces neonatal mortality and morbidity especially due to infections, among preterm babies in hospital with a weight of $\leq$ $2000 \mathrm{~g}$ and reduces risk of post-discharge mortality $[5,20,21]$. There is thus a probability that KMC also reduces the rates of community acquired infections among these infants. Furthermore, KMC also strengthens what has been referred to as "the attachment relationship" between the parents including fathers and their babies it is also important to note that all the babies were being exclusively breast fed [20]. This is in consonance with reports that show higher rates of exclusive breast feeding in babies who received KMC [21-23].

The challenges faced by mothers practicing KMC in this study have also been highlighted by other authors [24,25]. Lack of help with KMC practice and other obligations have been highlighted as a barrier to the practice of KMC [21-24]. Some of the mothers did have support in areas such as care for other children and help with household chores. The need for family support for mother-infant pairs practicing KMC cannot be over-emphasized. Authors have described KMC as both a restorative as well as an energy-draining experience [15]. A supportive environment has been described as facilitating the restorative experience, whereas obstacles in the environment seem to make the provision of KMC energy-draining for parents. Family and community support for the mother infant dyad cannot be over emphasized. In places where the practice is established, KMC usually starts in hospital with an adaptation process. During this process and post discharge, mothers/caregivers are trained, infants are monitored, and the mothers/caregivers are eventually absorbed into support groups or social peer networks [2]. Care is thereafter provided at home with follow up visits as needed. Such groups in addition to support from family and friends are necessary to improve practice.

Mothers can share the role of provider of the kangaroo position with others, especially the babies' fathers [2]. Less than half of the mothers in the study were aware that someone else could assist with $\mathrm{KMC}$ at home despite the fact that they had been counseled before and during the practice of $\mathrm{KMC}$ in the hospital. This may be attributed to either lack of adequate information given or that information was not well understood. This brings to light the fact that mothers need education and reeducation for information to have its full impact. Majority of those who knew they could be assisted however still preferred to do it themselves. Of the others, grandmothers rather than fathers were the most common assistants. This is not unusual as grandmothers do play a significant role in childcare in Nigeria. As part of education prior to discharge fathers and perhaps grandmothers too should be encouraged to get involved as $\mathrm{KMC}$ has been reported to strengthen bonding between the parents (father inclusive) and their baby [20]. Grand mothers are known to have a strong commitment to promoting the well-being of children, their mothers and families and do participate in child care in Africa [26].

The commonest reason given for termination of KMC was that the baby had become uncomfortable with the procedure. This is in consonance with the well documented fact that babies discharge themselves from KMC when they no longer need it [2]. Infants will reject permanent contact once they achieve regulation of their body temperature, at a median age of 37 weeks after conception other reasons included lack of support for domestic chores and uncomfortable sleeping positions [2]. The need for support has already been highlighted. Difficulty in sleeping with the infant in KMC position throughout the night has also been reported by other authors with the plausible reason that an uncomfortable sleeping position caused insufficient sleep [25]. 
The finding that all the mothers would recommend $\mathrm{KMC}$ to other mothers with low birth weight infants is similar to what has been reported in other studies [27,28]. This is perhaps a reflection of their satisfaction and the potential benefits derived from KMC. In the same vein majority of the mothers would gladly repeat KMC if they had another low birth weight infant. Studies comparing KMC mothers with those whose babies had conventional care showed higher levels of satisfaction amongst KMC mothers [16,21,24]. Some others who were silent on this were only hopeful that they would not have another such infant and looking forward to practicing it again may attract another LBW infant. Myths like this are not uncommon in Africa.

In conclusion, mothers are generally comfortable with $\mathrm{KMC}$ but need more education and support. Support groups and social networks are advocated to improve the practice.

\section{References}

1. Wardlaw T, Blanc A, Zupan J, et al. United Nations Children's Fund and World Health Organization. Low Birthweight: Country, Regional and Global Estimates. 2005.

2. Ruiz-Peláez JG, Charpak N, Cuervo LG. Kangaroo Mother Care, an example to follow from developing countries. BMJ 2004;329:1179-81.

3. Mwendwa AC, Musoke RN, Wamalwa DC. Impact of Partial Kangaroo Mother Care on Growth Rates And Duration Of Hospital Stay Of Low Birth Weight Infants At The Kenyatta National Hospital, Nairobi. East Afr Med J. 2012;89(2):53-8.

4. Rey ES, Martinez HG. Rational management of the premature infant. Bogotá, Colombia: Colombia Universidad Nacional. 1983:137-51.

5. Lawn JE, Mwansa J, Horta BL, et al. 'Kangaroo mother care'to prevent neonatal deaths due to preterm birth complications. Int J Epidemiol. 2010;39:144-54.

6. Charpak N, Ruiz-Palaez JG, Figueroa de CZ, et al. A randomized, controlled trial of kangaroo mother care:results of follow up at one year corrected age. Pediatrics. 2001;108(5):1072-9.

7. Affonso D, Wahlberg V, Persson B. Exploration of mother's reactions to the kangaroo method of prematurity care. Neonatal Netw. 1989; 7:43-51.

8. Charpak N, Riuz-Pelaez JG, Figueroa Z, et al. Kangaroo mother versus traditional care for newborn infants less than or equal to 2000 grams: a randomised controlled trial. Pediatrics 1997;100:682-9.

9. Charpak N, Riuz-Pelaez JG, Figueroa Z, et al. A randomised controlled trial of Kangaroo mother care: results of follow-up at one year of corrected age. Pediatrics 2001;108:1072-9.

10. World Health Organization. Reproductive Health. Kangaroo mother care: a practical guide. World Health Organization; 2003.

11. Cattaneto A, Davanzo R, Worku B, et al. Kangaroo mother care for low birthweight infants: a randomized controlled trial in different settings. Acta Pediatrica. 1998;87(9): 976-85.

12. Charpak N, de Calume ZF, Ruiz JG. "The Bogota declaration on kangaroo mother care": conclusions at the second international workshop on the method. Second International Workshop of Kangaroo Mother Care. Acta Paediatr 2000;89:1137-40

13. Charpak N, Ruiz-Peláez JG, Charpak YRM. Kangaroo Mother Program: an alternative way of caring for low birth weight infants? One year mortality in a two cohort study. Pediatrics. 1994;94(6):804-10.

14. Affonso D, Bosque E, Wahlberg V, et al. Reconciliation and healing for mothers through skin-to-skin contact provided in an American tertiary level intensive care nursery. Neonatal Netw. 1993;12(3):25-32.

15. Anderzen-Carlsson A, Lamy ZC, Eriksson M, et al. Parental experiences of providing skin-to-skin care to their newborn infant-Part 1: A qualitative systematic review. Int J Qual Stud Health Well-being. 2014;9(1):24906.

16. Legault M, Goulet C. Comparison of kangaroo and traditional methods of removing preterm infants from incubators. J Obstet Gynecol Neonatal Nurs. 1995;24:501-6.

17. Bell EH, Geyer J, Jones L. A structured intervention improves breast-feeding success for ill or preterm infants. Am J Matern Child Nurs. 1995;20:309-14.

18. World Health Organization. March of Dimes, The Partnership for Maternal, Newborn \& Child Health, \& Save the Children. Born too soon: The global action report on preterm birth WHO. 2012.

19. Conde-Agudelo A, Díaz-Rossello JL, Belizan JM. Kangaroo mother care to reduce morbidity and mortality in low birthweight infants. Cochrane Database Syst Rev. 2003;2(2):CD002771.

20. Tessier R, Cristo M, Velez S, et al. Kangaroo mother care and the bonding hypothesis. Pediatrics. 1998;102(2):e17.

21. Flacking R, Ewald U, Wallin L. Positive effect of kangaroo mother care on long-term breastfeeding in very preterm infants. J Obstet Gynecol Neonatal Nurs. 2011;40(2):190-7.

22. Ramanathan K, Paul VK, Deorari AK, et al. Kangaroo mother care in very low birth weight infants. Indian $\mathrm{J}$ Pediatr. 2001;68(11):1019-23.

23. Uwaezuoke SN. Kangaroo mother care in resource-limited settings: implementation, health benefits, and costeffectiveness. Res Rep Neol. 2017;7:11-8.

24. Seidman G, Unnikrishnan S, Kenny E, et al. Barriers and Enablers of Kangaroo Mother Care Practice: A Systematic Review. PLoS One. 2015;10(5):e0125643.

25. Blomqvist YT, Frölund L, Rubertsson C, et al. Provision of Kangaroo Mother Care: supportive factors and barriers perceived by parents. Scand J Caring Sci. 2013;27(2): 345-53.

26. Van Zyl M. The Ekwendeni Agogo Approach: Grandparents as agents of change for newborn survival. Save the Children. 2010.

27. Nguah SB, Wobil PNL, Obeng R, et al. Perception and practice of Kangaroo Mother Care after discharge from 
hospital in Kumasi, Ghana: A longitudinal study. BMC Pregnancy Childbirth. 2011;11:99.

28. Hill Z, Tawiah-Agyemang C, Manu A, et al. Keeping newborns warm: beliefs, practices and potential for behavior change in rural Ghana. Trop Med Int Health. 2010;15(10):1118-24.

*Correspondence to:

Peace Opara

University of Port Harcourt Teaching Hospital

Port Harcourt,

Nigeria

Tel: 706-750-2572

E-mail: peaceibo@yahoo.com.au 